\title{
Adaptive Token Polling MAC Protocol for Wireless Underwater Networks
}

\author{
Gunilla E. Burrowes \\ School of Electrical Engineering and Computer Science \\ University of Newcastle \\ Newcastle, Australia \\ Email: gunilla@ieee.org
}

\author{
Jamil Y. Khan \\ School of Electrical Engineering and Computer Science \\ University of Newcastle \\ Newcastle, Australia \\ Email: jamil.khan@newcastle.edu.au
}

\begin{abstract}
The opportunities and applications for a large group of coordinating autonomous underwater vehicles (AUV) is exciting and provides greater exploration opportunities than fixed sensor nodes or using a single AUV. The work presented in this paper is the development of a Medium Access Control Protocol for a cluster of small autonomous vehicles working in a centralized network topology. Acoustic communication links are typically used for underwater operations, however are substantially limited by the physics of acoustic propagation which require essentially new scheduling protocols to those being used and developed for packet radio networks.

This paper provides a discussion and overview of the essential issues for developing a new MAC protocol for a cluster of closely operating underwater vehicles and presents some performance results for a proposed Adaptive Token Polling Protocol.
\end{abstract}

\section{INTRODUCTION}

Applications for groups of small underwater autonomous vehicles are beginning to be realized as the technological developments of these vehicles are becoming a reality [1]. The opportunities and applications for a large group of coordinating autonomous underwater vehicles (AUV) is exciting and provides greater exploration opportunities than fixed sensor nodes or using a single AUV. Applications include data collection such as scientific, environmental or pollution detection, exploration of underwater resources, monitoring of equipment such as platforms and cables, as well as adaptive sensing of chemical leaks or biological phenomena such as oil leaks or phytoplankton concentrations [2], [3] and in search and find mission scenarios. There are also numerous military applications that would benefit from multiple AUV systems, including mine hunting and reconnaissance, and monitoring of harbours and dock areas for security. [3]

The development of a Medium Access Control (MAC) protocol for an underwater acoustic communication network will focus on sampling and searching operations, which accounts for many of the potential beneficial applications suggested. These applications are currently not being implemented extensively due to not only the technical limitations but also the commercial constraints which are bound to the current technology capability [1]. As the development of small inexpensive underwater AUV's that can work cooperatively together become a practical reality these application scenarios will grow significantly as the breadth, scope and need for knowledge of underwater conditions and environments continues to grow. Sampling applications, in general, require delay-insensitive data with a focus more on the collection and storage of the sensor data until it can be uploaded and analysised. While searching applications require delay-sensitive data as analysis of that data is required to lead to future decisions of the swarm. In both cases, however, delay-sensitive data is required of the navigational information that is required by the swarm to avoid collision with obstacles as well as with each other.

The focus for this paper is on the development and performance of a MAC protocol for a short range underwater sensor network. The design is based on an acoustic physical layer and is designed to make best use of the transmission medium. In this paper we propose a contention free deterministic adaptive token polling protocol. The paper begins by reviewing the unique requirements of a MAC protocol for underwater acoustic communication and some recent work that focuses on short range mobile operations. Section V and VI summaries the network topology and protocol before introducing the analysis and performance of the Adaptive Token Polling algorithm in Section VII.

\section{UNDERWATER VS TERRESTRIAL WIRELESS ENVIRONMENTS}

For clusters, and eventually swarms, of multiple mobile vehicles to be practical, they will require the ability to work cooperatively by exchanging location and movement information as well as the sensor data that they are collecting. To some extent these are similar problems faced by the terrestrial wireless ad-hoc and sensor networks, however information exchanges becomes more critical for underwater vehicles as they are operating without GPS and usually navigating through unknown environments. This location information is also required for information forwarding purposes.

The first challenge for underwater communication networks is that the channel itself presents very different and unique characteristics compared to the terrestrial channel and the characteristics of working in seawater include absorption, reverberation, noise and multipath. In particular, acoustic communication which is the typically mechanism used for underwater operations, inherently introduce a very high propagation delay 
which is $0.67 \mathrm{~ms} / \mathrm{m}$ whereas a radio link introduces a propagation delay of $3.33 \mathrm{~ns} / \mathrm{m}$ in air. RF, underwater, even at low frequencies suffers from extreme attenuation due to conductive seawater and high rates of absorption that has predominately eliminated its use for long range underwater communications. In addition, underwater communication channel characteristics change more dynamically than in terrestrial channels due to its noise and thermal profiles [4]. Thus with significantly longer propagation delays and low bit rates, the acoustic underwater channel is fundamentally different from a radio based packet network and as such require essentially new scheduling MAC protocols.

\section{ReQuirements of An Acoustic MAC Protocol}

Thus requirements of a MAC protocol in an underwater acoustic network must take into account the high propagation delay environment and thus the affect that this has on the delay in message and control signal transmissions. Without consideration of this delay, the reliability of the transmissions would be severely reduce due to the potential interference and increase in errors particularly at the receiving nodes. The result of this leads to the need for retransmissions and therefore lower throughput and higher energy consumption. The delay and low bit rate environment leads to the necessity to minimise the overheads compared with the size of the message but this can have implications on reliability and therefore the efficiency of the overall algorithm.

Energy consumption is a critical factor for AUV's as the opportunity to recharge during a mission is not practical and therefore a protocol must optimise its transmission and scheduling techniques. The MAC protocol design can improve the energy consumption by appropriately designing the transmission and sleep cycles.

\section{RELATED WORK}

There are very few research projects that have investigated protocols for very short range wireless underwater communication (in the order of $100 \mathrm{~m}$ ) as the vehicle technology has not been available for this need. One of the few projects found to have done this is by MIT and Woods Hole [5] that developed a TDMA (Time Division Multiple Access) based protocol for a concurrent mapping project which used multiple AUV's to build maps of underwater areas at internode distances of $100 \mathrm{~m}$. TDMA was chosen here to avoid the need for simultaneous multiple user signal detection and was able to be optimised for a fix number of nodes with regular and known data collection. It was concluded that as the coverage area increased and/or the number of nodes increased, TDMA would no longer be an efficient technique to use in terms of both link utilization and power conservation. However, good results were found for a network of 5 nodes in a $1 \mathrm{~km}^{2}$ region.

Investigation into the use of TDMA as a sub-cluster or short range scheduling protocol has also been proposed for a multi-cluster ad-hoc mobile underwater acoustic network. In this project the topology used was a distributed node system with the focus of the work on the use of CDMA (Code division Multiple Access) as a inter cluster approach that can provide efficient scalability by the spatial reuse of channel resources. Salva-Garau and Stojanovic [6] designed a TDMA protocol as the intra-cluster protocol using the lowest-identifier clustering algorithm (LIDCA) for slot priority determination. The paper did not separate the analysis of the results for the separate benefits of TDMA and CDMA but looked at the system results as a whole. It showed that with smaller cluster sizes the TDMA cycle time is reduced due to fewer slots required and thus frame size shorter, giving an advantage of higher transmission rates overall which infers that TDMA is very much limited by transmission range. However, reducing frame size means that the ratio of the overheads, which is a fixed length management slot, to transmitted data becomes less efficient. The authors also showed that through the spatial reuse of CDMA codes via the clustering structure, there was an improvement in the efficiency of network resources. The cluster concept with different intra and inter cluster protocols was shown to optimise the overall system efficiencies by being able to design for the two very different communication needs and conditions.

More recent work in the ACME project (Acoustic Communication network for Monitoring of Environment in coastal areas) sponsored by the European Union [7] has developed the ACMENet protocol which is a polling based TDMA algorithm for a master-slave topology. This research demonstrated that having a central controller provided the opportunity to increase the efficiency of the TDMA protocol by using a polling request process to optimise the use of time slots. The controller was also used in a network management function as a rule based decision maker to improve efficiency of power consumption within the node and was able to select the optimum modulation type as a global parameter of the system. Included as part of this work were extensive sea trials that highlighted the problematic nature of the underwater environment and the detrimental effects on the performance of the protocol. High packet loss ratios and link outages were recorded due to the high acoustic noise generated by shipping traffic. Thus the robustness of the protocol and error correction techniques will be important to consider.

\section{Network Topology}

The proposed centralised two-tiered network topology of underwater mobile vehicles is shown in Fig. 1. where the Slave Nodes form into clusters with a single Master Node. These clusters are made up of numerous Slave Nodes which can vary in number as Slave Nodes move from cluster to cluster. The optimum number of Slave Nodes in a cluster will vary with channel and application conditions and will be studied in more detail in future work. The Slave and Master Nodes are mobile autonomous vehicles with the more sophisticated Master vehicle sitting above the cluster and controlling the work of the Slave Nodes. The Slave Nodes are an identical set of simple sensor data collection vehicles that are designed for very low processing power, and will be relatively cheap to manufacture and use. The cluster topology has the advantage 
of forming repeating clusters for scalability of the system and these groups of cluster will form a swarm. The work presented in this paper focuses on the development of a MAC protocol to support packet transmissions in a two tier Master-Slave based underwater sensor network application.

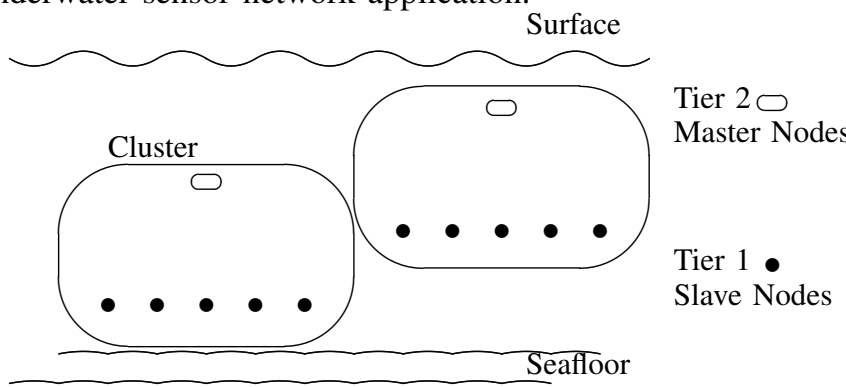

Fig. 1: Centralised Cluster Network Topology for AUV's

In these clusters all nodes will be assumed to be mobile at around 2 knots $(1 \mathrm{~m} / \mathrm{s})$, with planned senor data collection done periodically, at every $10 \mathrm{~m}$ or every $10 \mathrm{~s}$ by each of the Slave Nodes. This data will be evaluated by the Slave Nodes and compared to its previous data entry and then uploaded to the Master Node only when there is some predetermined change in the data. The Slave Nodes will also be using DVL (Doppler Velocity Log) and compass data collection for navigational purposes which will be collected and evaluated once per second but in the same fashion as the sensor date will only be sent on to the Master Node if a change has occurred. This evaluation will not substantially increase the complexity of the Slave Node or power consumption but will save on unnecessarily communication in this limited channel environment. The Master Node will use this information to create a map of the Slave Nodes location and direction of movement for control of the cluster. The cluster topology and the location and movement data, will also assist in future operations when more clusters are added and routing protocols will be required to be implemented to control the swarm of vehicles.

This research is thus focused on short range mobile applications with communication requirements between vehicles that could vary from a constant stream of data in some situations to bursty traffic in others, which suggests some form of adaptive protocol due to the variability of data quantity.

Also, as the application is built based on a centralised network, with master-slave architecture, a polling protocol will be used. Polling protocols have advantages in underwater systems compared to TDMA as there is less reliance on synchronization which TDMA requires and can more easily improve transmission efficiency as slots do not need to be held open if there is no data to send. Due to the propagation delay, a token polling operation rather than the more conventional rollcall polling will be used. Conventional polling has an overhead of two times the propagation delay compared to the token which does not have to wait for a reply.

Token polling requires that each Slave Node broadcasts their reply so that all nodes in the system keep track of current transmitting status. The token is attached to the end of the final data packet sent by the currently transmitting Slave Node and includes the next Slave Node's address to indicate to them that they have the right to use the channel.

\section{Adaptive Token Polling Algorithm}

\section{A. Cycle Process}

The Adaptive Token Polling MAC Protocol proposed in this work is based on a centralised hub polling algorithm [8]. It is an adaptive algorithm in that the slot does not stay open if there is no data and would immediately passed on the token instead.

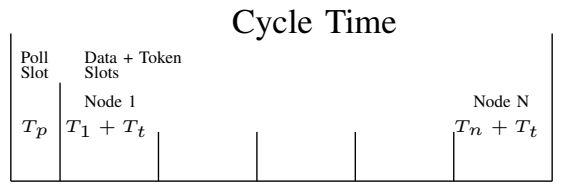

Fig. 2: Adaptive Token Polling Cycle

A typical single cycle is illustrated in Fig. 2 and has two phases, polling and data transmission. The number of Slave Nodes can vary and is defined here as $\mathrm{N}$ Slave Nodes per cluster. Each cycle begins with a Poll message where the Master Node broadcasts to all the Slave Nodes in its cluster, a poll of length $V_{p}$ bits which has a transmission time of $T_{p}$ sec. This poll provides the synchronization and management of the cluster as well as defines the nodes in its cluster by the address structure used in its header. The poll will also provide the token which will identify and acknowledge the first Slave Node permitted to send data to the Master Node. The transmission sequence through the Slave Nodes is based on LIDCA so that the Master Node passes it's token to the Slave Node with the lowest ID in the cluster and then sequentially in ascending order of node ID value (represented by $n$ ). When the lowest ID Slave Node receives the token from the Master, it can begin transmission of its next queued packet of data (control and sensor) of variable length $X_{n}$ bits plus the length of the token $V_{t}$ bits which have a transmission time of $T_{n}$ and $T_{t}$ sec respectively. If there is no data queued then the Slave Node will immediately send on just the token. Thus the cycle time may vary depending on the size of the data packets queued and the number of slots which have data packets to send.

\section{B. Structure of Frames}

The poll, token, and data frame structures are shown in Fig. 3. Their design is based on the IEEE802.15.4 standard but optimised to reduce transmitting any unnecessary information. This has reduced the polling and token only messages to 12 bytes, and if a full control and sensor data packet is to be sent, a slot packet size is 192 bytes.

Each of the frames begins with a synchronization preamble and finishes with a FCS error correction field. All three frames also carry the list of Slave Node addresses which is made up of one byte, with the first 4 bits giving the cluster number (CCCC) and the second 4 bits the ID number of the node. The node ID CCCCO000 will always represent the Master Node of 
that cluster which means that this address system will provide up to 15 nodes per cluster and 225 vehicles in a 15 cluster swarm. The token byte is also given in all frames to identify the cluster and next node address.

\begin{tabular}{|l|l|l|l|l|l|l|l|l|}
\hline Field & Preamble & \multicolumn{3}{|c|}{ Header } & Token & \multicolumn{2}{|c|}{ Data } & Error \\
\hline $\begin{array}{l}\text { Information } \\
\text { Type }\end{array}$ & Synch & $\begin{array}{l}\text { List of } \\
\text { Addresses }\end{array}$ & $\begin{array}{l}\text { Tx Length } \\
\text { Field }\end{array}$ & $\begin{array}{l}\text { Poll } \\
\text { Managemen }\end{array}$ & Token & Control & Sensor & FCS \\
\hline Poll & 4 & 1 & - & 2 & 1 & - & - & 4 \\
\hline Token & 4 & 1 & 2 & - & 1 & - & - & 4 \\
\hline Data & 4 & 1 & 2 & - & 1 & $0-80$ & $0-100$ & 4 \\
\hline
\end{tabular}

Fig. 3: Frame Structure for Poll ( $V_{p}=12$ Bytes),Token $\left(V_{t}=12\right.$ bytes), Data with inbuilt Token $\left(X_{n}+V_{t}=12\right.$ to 192 bytes $)$

The Poll frame's other information field is a 2 byte Management message which will provide speed and directional changes to any individual Slave Node from the Master. The Poll Management bytes will also include the ACK for the previous cycles which will provide feedback to the Slave Nodes on their previous attempt. This single ACK approach is much more time efficient than a system where all messages require a response.

The data and token frames do not have the 2 byte Poll Management field but instead has a Transmission Length Field of equivalent size. This field provides the details of the length of the data portion of that frame which will be used to instruct other nodes in the cluster on how long nodes can sleep.

\section{ANALYSIS AND PERFORMANCE}

\section{A. Delay Analysis}

The analytical model is presented to evaluate the packet delay and cycle performance for the adaptive polling protocol. The model is based on the M/G/1 queuing model where we have assumed that packets are generated at each node according to the Poisson process with rate $\lambda$. The performance measures evaluated here include the average waiting time, average cycle time, average number of packets in a node queue, and average transfer delay.

In an average cycle time $\bar{T}_{c}$ there is expected to arrive $N_{c}$ packets. Using Little's Theorem, the mean number of arrivals $\mathrm{A}(\mathrm{t})$ across all nodes in time $\mathrm{t}$, is $\mathrm{E}[\mathrm{A}(\mathrm{t})]=N \lambda t$. Thus $N_{c}=$ $\mathrm{E}\left[\mathrm{A}\left(\bar{T}_{c}\right)\right]=N \lambda \bar{T}_{c}$.

The time for one cycle can also be found by adding up the time for each of the time slots within it. Thus, following the structure illustrated in Fig. 2, the average cycle time is $\bar{T}_{c}=T_{p}+N_{c} \bar{T}_{n}+N T_{t}+(N+1) \tau_{\max }$ where $\tau_{\max }$ is the propagation time to the furthest node. Substituting $N \lambda \bar{T}_{c}$ for $N_{c}$ and rearranging for $\bar{T}_{c}$ gives the average cycle time:

$$
\bar{T}_{c}=\frac{T_{p}+N T_{t}+(N+1) \tau_{\max }}{1-N \lambda \bar{T}_{n}}
$$

To derive the expression for the average transfer delay or average time the packet is expected to spend in the system $\bar{D}_{t}$, the average waiting time a message has to spend in a queue before it starts its transmission $\bar{W}_{t}$ needs to be calculated. Assume, for example, an arbitrary packet arrives at a Slave Node. The time it has to wait in the queue is the remaining time of the cycle which is currently in operation plus the sum of the number of cycles that are required to empty that nodes queue so that it is next to proceed from that node. The first term can be estimated in the steady state to be half the cycle time: $\frac{\bar{T}_{c}}{2}$. The second term uses Little's Theorem above to find the expected number of packets waiting in a nodes queue: $\frac{E\left[N_{c}\right]}{N}=\lambda \bar{W}_{t}$. Thus, the average expected waiting time is given by

$$
\bar{W}_{t}=\lambda \bar{W}_{t} \bar{T}_{c}+\frac{\bar{T}_{c}}{2}
$$

rearranging for $\bar{W}_{t}$ and substituting the solution for $\bar{T}_{c}$ from (1):

$$
\bar{W}_{t}=\frac{T_{p}+N T_{t}+(N+1) \tau_{\max }}{2-2 N \lambda \bar{T}_{n}-2 \lambda\left(T_{p}+N T_{t}+(N+1) \tau_{\max }\right)}
$$

and thus the average transfer delay a packet experiences in the system is:

$$
\bar{D}_{t}=\bar{W}_{t}+\bar{T}_{n}+\tau_{\max }
$$

And finally, determining the number of packets in a station queue is thus:

$$
\bar{N}_{c}=\lambda \bar{W}_{t}
$$

\section{B. Results}

The following results are obtained from MATLAB simulations using the M/G/1 queuing model presented in Section VII $\mathrm{A}$ and are based on ideal channel conditions. Processing time has not been included and the queue buffer for all nodes has been assumed to have infinite message storage capacity. This work has been presented to set out the limits of these system parameters for this Adaptive MAC protocol as a benchmark to use in future developments of more realistic models planned for this research project.

The base system parameters, for a typical industry application, are; an average 90 byte payload packet (102 byte Data Frame), arrival rate of 0.2 packets/s/node, 5 nodes per cluster and an internodal range of $50 \mathrm{~m}$ using a $7000 \mathrm{bps}$ channel transmission rate. This transmission rate is based on the LinkQuest Acoustic Modem UWM1000 [9] specification. It is a two-way, half duplex acoustic modem designed for shorter distances and is specified to have a $17.8 \mathrm{kbps}$ acoustic link and 7000bps payload data rate at a working range of $350 \mathrm{~m}$.

Using these parameters we evaluate the protocol by first varying the arrival rate (packets per second per node) to investigate the average packet delay per Slave Node given by Eqn 4. as shown in Fig. 4. Looking first at our proposed industry application 'average' case, of 5 nodes, with an average 102 byte data frame per node, $50 \mathrm{~m}$ range and arrival rate of 0.2 packets/s/node the average packet delay per node is less than $0.5 \mathrm{sec}$. This delay and arrival rate would not cause any queuing of frames within each node. In fact the arrival rate could increase to over 1 packet/s/node before nodes may expect to have queued data frames. Should nodes move or drift apart within the cluster, the effect of propagation delay can clearly be seen with the system needing to work with lower arrival rates for similar packet delays. Similarly, if the number of 
nodes increase within the cluster, from 5 to 9 nodes shown in Fig 4., there are limits to the quantity of data per node that can be transmitted within the system. This is due to the substantial increase in cycle time required as more nodes would mean more slots required within each cycle. Fig. 4 also demonstrates the change in arrival rate when the size of the payload increases. These results are consistent with the balance expected in data quantities required to be transmitted. That is, if twice as much data is incorporated in one frame, that frame would need to arrive half as often to maintain similar average packet delays.

Fig. 5 presents the analysis of Eqn. 5, the average queue expected in each node, when the payload per node in bytes per packet is increased. These results mirror the conclusions found in Fig 4. where the balance between arrival rate and payload size needs to be maintained when fixing other parameters in the system. It can be seen that once conditions are such that more than one packet is in a node queue, than the queue will continue to build up very swiftly unless conditions can be eased during operation.

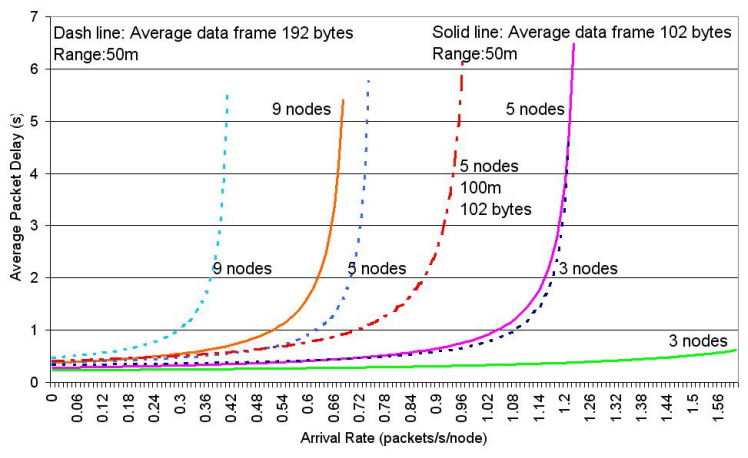

Fig. 4: Average Packet Delay vs Arrival Rate with node and range variations

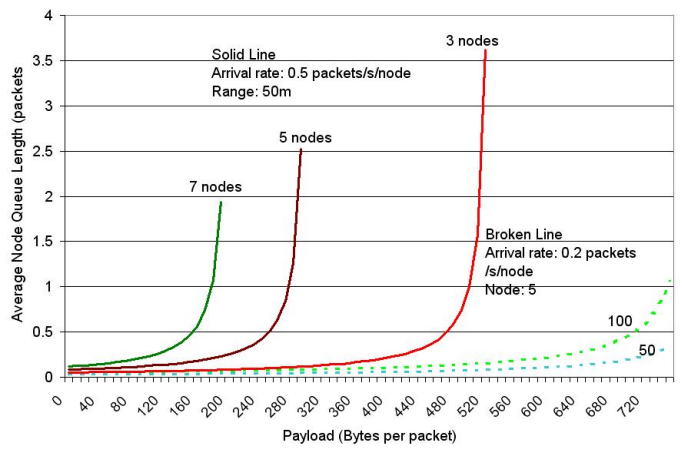

Fig. 5: Average Node Queue Length vs Payload with arrival rate, node and range variations

Thus the number of nodes per cluster is a critical parameter and will need to be carefully planned for within the protocol. This may mean that not only should the protocol be adaptive to the variability of data frame size but also feedback dynamically to the on board processor to adjust arrival rate in each node. In fact, arrival rate verse packet size needs to be monitored and adjusted to ensure that the average packet delay does not become unacceptable for the particular application requirements.

\section{CONCLUSION}

The background to this research has grown from the current developments in terrestrial wireless ad-hoc and sensor network communication technology and their adaptation and implementation in underwater environments. The ocean environment is significantly different to the terrestrial communication channel and therefore require fundamentally different solutions.

This paper has presented a new Medium Access Control protocol for intra-cluster communication for a two-tiered centralised AUV network. Performance analysis of this protocol has been presented with results that show the potential to operate clusters of AUV's in close proximity to each other and exchange navigational and sensor data in an acceptable time frame for a search operation. This work has set the foundation and best case scenario's for this system operation. The next stages of this research will be to extend the protocol with sleep cycles and evaluate the energy consumption during an operational cycle. The development of an appropriate underwater communication channel model in which to test the protocol is also imperative.

This is clearly a very challenging research area and one that is only just emerging as a field in its own right. The extreme limitations which both salt water itself and the ocean environment place on what are already difficult problems in terrestrial work means that substantially different solutions need to be found.

\section{ACKNOWLEDGMENT}

The authors would like to thank ATSA Defense Services for their support of this research project.

\section{REFERENCES}

[1] R. Wernli, "Auv commercialization-who's leading the pack?" OCEANS 2000 MTS/IEEE Conference and Exhibition, vol. 1, pp. 391-395, 2000.

[2] J. Heidemann, W. Ye, J. Wills, A. Syed, and Y. Li, "Research challenges and applications for underwater sensor networking," in Proceedings of the IEEE Wireless Communications and Networking Conference. Las Vegas, Nevada, USA: IEEE, April 2006, pp. 228-235. [Online]. Available: http://www.isi.edu/ johnh/PAPERS/Heidemann06a.html

[3] D. Pompili, T. Melodia, and I. Akyildia, "Routing algorithms for delayinsensitive and delay-sensitive applications in underwater sensor networks," in Proceedings of MobiCom. Los Angeles, California, USA: ACM, September 2006, pp. 298-309.

[4] J. Preisig, "Acoustic propagation considerations for underwater acoustic communications network development," in Conference. WUWNet, 2006.

[5] M. Stojanovic, L. Freitag, J. Leonard, and P. Newman, "A network protocol for multiple auv localization," in Proc. IEEE OCEANSO2 Conference. Proc. IEEE OCEANS02 Conference, 2002, pp. 604-611.

[6] S. Salva-Garau and M. Stojanovic, "Multi-cluster protocol for ad hoc mobile underwater acoustic networks," in MTS/IEEE OCEANS, 2003.

[7] G. Acar and A. E. Adams, "Acmenet: An underwater acoustic sensor network protocol for real-time environmental monitoring in coastal areas," IEE - Radar Sonar Naviagation, vol. 153, no. 4, pp. 365-380, 2006.

[8] W. C. Chan, Performance Analysis of Telecommunications and Local Area Networks. Boston/London: Kluwer Academic Publishers, 2000.

[9] L. Inc., SoundLink Underwater Acoustic Modems, High Speed, Power Efficient, Highly Robust, LinkQuest Inc., http://www.link-quest.com/, 2008. 